\title{
The effect of season on aspects of in vitro embryo production in sub-fertile beef cows
}

\author{
J.M. Rust $^{1 \#}$, D.S. Visser ${ }^{2}$, J.E. Venter, M.P. Boshoff, S. Foss and J.P.C. Greyling ${ }^{3}$ \\ ${ }^{1}$ Döhne ADI, Stutterheim, South Africa; \\ ${ }^{2}$ Animal Production Institute, Irene, South Africa; \\ ${ }^{3}$ University of the Free State, Bloemfontein, South Africa
}

\begin{abstract}
This study was conducted to investigate the possible effect of season on in vitro embryo production (IVEP) in sub-fertile beef cows. Forty beef (40) cows of different breeds and parities were used in a trial conducted as part of a commercial embryo production programme. Animals were subjected to a once a week ultrasound guided oocyte recovery procedure (OPU) and all oocytes were entered in a commercial IVEP programme. A significant difference in follicular populations could be established for different months of the year. As for recovery rate, oocyte quality and embryo production, no significant differences could be established. However, the embryo production per OPU session per cow showed a definite seasonal pattern with low production during hotter months, a steady increase during the colder months and a peak in spring. This information should be helpful in the future planning of IVEP programmes for fertility-impaired cows.
\end{abstract}

Keywords: In vitro embryo production, OPU, season, fertility, assisted reproduction

${ }^{\#}$ Corresponding author. E-mail: jean.rust@gmail.com

\section{Introduction}

It is an established fact that season can influence the reproduction performance of both the Bos indicus and Bos taurus cattle breeds (Zeitoun et al., 1996). In Bos indicus cattle it was found that the cows exhibited better fertility during the spring and summer months, compared to the autumn and winter (Harrison et al., 1982; Rhodes et al., 1982; Bastidas \& Randel, 1987; Randel, 1994). In Bos indicus cows this limiting effect seems to be more pronounced during the colder months, whereas in the Bos taurus, the effect seems more pronounced during the warmer months (Zeitoun et al., 1996).

The effect of season on in vitro embryo production (IVEP) from abattoir material and in vivo derived oocytes has been previously investigated and a definite influence of season on embryo production has been recorded. The same fertility pattern normally recorded during conventional reproduction can also be found when multiple ovulation and embryo transfer (MOET) or IVEP results are considered (Rutledge et al., 1999). The effect of season on IVEP in seasonal breeders such as for example sheep has also been demonstrated (Mitchell et al., 2000).

In animals that experience some reproductive dysfunction and where the possibilities of producing embryos in vitro are limited, it is thus even more important that these procedures should not be attempted during periods where the chances of success are further limited by external factors such as season. The elements of IVEP such as ovarian follicular populations, oocyte recovery rates, oocyte quality and embryo production rates are important aspects of any in vitro embryo production system (Rutledge et al., 1999). In this study the effect of season on the IVEP parameters carried out over a period of four years were investigated.

\section{Materials and Methods}

Cows of various breeds and parities $(n=40)$ used in this trial were utilized for commercial embryo production purposes. These animals were from different beef cattle breeds (Simmental, Bonsmara, Santa Gertrudis, Romagnola and Brahman) and varied in age between two and 19 years. The average body condition score (BCS) of these animals was 2.5. The only common denominator was the fact that all these animals were classified as being unfit for further reproduction in the herd of origin. 
Prior to acceptance in the laboratory IVEP programme, all cows were rectally and ultrasonically evaluated (Pieterse et al., 1988) to determine whether the ovarian structures and functions were normal (development of at least one normal follicle during an oestrous cycle). Data were recorded over a 4-year period (2000 to 2003).

The ultrasound guided oocyte retrieval (OPU) procedure (Pieterse et al., 1988) was performed on a weekly basis on a fixed day of the week. This was done to keep the interval (number of days) between oocyte retrievals constant. An ultrasound apparatus (Pie Medical 200 Vet, Pie Medical, Holland) with a 5/7.5 MHZ sector scanner was utilized for ovary visualization. This ultrasound apparatus was equipped with a vaginal biopsy device and a dorsally mounted needle guide, specifically designed for the bovine OPU procedure. A $40 \mathrm{~mm}(20 \mathrm{G})$ disposable needle was used for follicular puncture and oocyte aspiration. The needle was connected to a disposable IVF set (tube set specifically for oocyte retrieval) (Pie Medical, Holland) connected to a $15 \mathrm{~mL}$ Falcon tube which in turn was connected to an aspiration vacuum pump (Cooke, Australia) - set at a constant vacuum pressure of $60 \mathrm{~mm} \mathrm{Hg}$. The Falcon collection tube was placed in a heating block (Cooke, Australia) set at a constant temperature of $35^{\circ} \mathrm{C}$. All visible follicles $>2 \mathrm{~mm}$ on both ovaries were aspirated, until no more follicles of this specific size were visible. The aspiration circuit was flushed at regular intervals with a PBS collection medium and the aspiration needle was replaced during collection whenever effective and smooth penetration of the ovary could no longer be achieved. All recovered oocytes were subjected to a standardized commercial IVEP protocol.

Parameters that were recorded included: the ovarian follicular populations (number of follicles $>2 \mathrm{~mm}$ ), oocyte recovery rate (number of oocytes recovered as a percentage of observed follicles), oocyte quality (as described by Leibfried \& First, 1979) and the number of embryos produced on a monthly basis over the four year period. Oocyte quality was defined according to the following criteria:

Grade A: These included all normal size oocytes, with a dense cumulus and a homogenous even-coloured ooplasm;

Grade B: The ooplasm of these oocytes were heterogeneous, granular, lightly

coloured and did not fill the zona pellucida (ZP);

Grade C: These included all the abnormally small oocytes.

The aspirated bovine oocytes were matured for a period of $24 \mathrm{~h}$. The maturation medium consisted of a modified TCM 199 medium (Gibco, Auckland, New Zealand), supplemented with FSH, LH, epidermal growth factor (EGF) and 10\% foetal calf serum (FCS). Incubation was performed in a Thermo Forma incubator at $39{ }^{\circ} \mathrm{C}, 5 \% \mathrm{CO}_{2}$ in air at saturated humidity which was achieved by placing a tray filled with water inside the incubator (D.S. Visser, 2003; ARC Animal Improvement Institute, personal communication).

After oocyte maturation (24 h incubation), the presumptive metaphase ll oocytes or ova were rinsed vigorously in handling medium (PBS medium, Sigma), supplemented with $1 \%$ newborn calf serum (NBCS) and heparin at a rate of $0.0183 \mathrm{~g} / \mathrm{L}$ in order to remove the excessive granulosa cells. This handling medium was used during all phases when ova or embryos were handled under normal atmospheric conditions. The mature ova were then rinsed once in Tyrode's lactate medium, supplemented with amino acids and bovine serum albumin (BSA) and transferred into Nunc multi-wells, containing the same medium, but further supplemented with heparin $(0.0183 \mathrm{~g} / \mathrm{L})$ (insemination medium)

A dose of bull semen $\left(0.25\right.$ or $0.5 \mathrm{~mL}$ straw) was thawed at $35^{\circ} \mathrm{C}$ for 30 seconds, before being gently overlaid on a pre-prepared discontinuous Percoll gradient consisting of $90 \%$ and $45 \%$ isotonic fractions in a test tube. The semen was then centrifuged at $700 \mathrm{G}$ for 30 minutes, the supernatant discarded and the sperm containing pellet re-suspended in $5 \mathrm{~mL}$ of the insemination medium. This was followed by a second centrifugation at $300 \mathrm{G}$ for $6 \mathrm{~min}$. The supernatant was again discarded and the concentration of the washed live spermatozoa in the resultant pellet was determined with the aid of a Makler counting chamber (D.S. Visser, 2003; ARC Animal Improvement Institute, unpublished data). After dilution of the sperm pellet with insemination medium, a final insemination dose of $1 \times 10^{6}$ per mL live sperm was used for insemination. The insemination dose was standard, regardless of the number of oocytes to be fertilized. Oocytes and sperm were then co-incubated for $24 \mathrm{~h}$ to complete the fertilization process (D.S. Visser, 2003; ARC Animal Improvement Institute, unpublished data). 
The presumptive zygotes were washed twice in handling medium $24 \mathrm{~h}$ after insemination. Two days before this stage, micro droplets $(2 \mu \mathrm{L})$ of embryo culture medium, overlaid with inert mineral oil (Sigma), were prepared and incubated $\left(39^{\circ} \mathrm{C}\right)$. Incubation was performed in a Thermo Forma incubator at $39{ }^{\circ} \mathrm{C}, 5 \%$ $\mathrm{CO}_{2}$ in air at saturated humidity which was achieved by placing a tray filled with water inside the incubator (D.S. Visser, 2003; ARC Animal Improvement Institute, personal communication).

The embryo culture medium consisted of CR2aa (aa = essential and non-essential amino acids) medium (Sigma) supplemented with 10\% FCS. Granulosa cells were added to the droplet to form a monolayer of cells in order to establish a co-culture system. Presumptive zygotes were then washed once in the embryo culture medium and then added to the micro droplets. Embryo culture was continued over a period of seven days, during which $50 \%$ of the culture medium was replaced with pre-equilibrated, fresh medium on days 3 and 6.

Data were analyzed by means of a one way ANOVA analysis of variance (SAS, 1986). Probability values $<0.05$ were considered to be statistically significant. The correlation co-efficient between the two data sets was determined by using the standard formula (statistical software for MS Excel) to determine the correlation co-efficient (r) between the two ranges of values.

\section{Results}

The mean ( \pm s.e.) follicular populations, number of oocytes recovered and oocyte recovery rate are set out in Table 1.

Table 1 The mean ( \pm s.e.) ovarian follicular development, number of oocytes recovered and recovery rate following oocyte recovery procedure (OPU) from fertility impaired cows over a 4-year period

\begin{tabular}{lcccc}
\hline $\begin{array}{c}\text { Month of } \\
\text { the year }\end{array}$ & $\begin{array}{c}\text { OPU } \\
\text { sessions } \\
(\mathrm{n})\end{array}$ & $\begin{array}{c}\text { Number } \\
\text { of } \\
\text { follicles }\end{array}$ & $\begin{array}{c}\text { Number of } \\
\text { oocytes } \\
\text { recovered }\end{array}$ & $\begin{array}{c}\text { Oocyte recovery } \\
\text { rate } \\
(\%)\end{array}$ \\
\hline January & 39 & $2.7^{\mathrm{a}} \pm 1.3$ & $1.4 \pm 1.2$ & $50.9 \pm 37.9$ \\
February & 64 & $3.3^{\mathrm{b}} \pm 2.4$ & $1.4 \pm 1.6$ & $39.5 \pm 37.2$ \\
March & 44 & $3.0^{\mathrm{b}} \pm 1.7$ & $1.4 \pm 1.4$ & $45.0 \pm 38.5$ \\
April & 22 & $2.6^{\mathrm{a}} \pm 1.8$ & $0.8 \pm 1.0$ & $33.7 \pm 38.0$ \\
May & 23 & $4.1^{\mathrm{b}} \pm 2.7$ & $1.8 \pm 1.9$ & $44.4 \pm 36.1$ \\
June & 64 & $3.2^{\mathrm{b}} \pm 1.6$ & $1.3 \pm 1.5$ & $36.4 \pm 35.6$ \\
July & 42 & $3.4^{\mathrm{b}} \pm 1.7$ & $1.5 \pm 1.5$ & $41.8 \pm 35.6$ \\
August & 29 & $3.5^{\mathrm{b}} \pm 1.6$ & $1.6 \pm 1.4$ & $46.8 \pm 36.2$ \\
September & 27 & $4.3^{\mathrm{c}} \pm 2.2$ & $1.9 \pm 1.7$ & $41.0 \pm 33.4$ \\
October & 60 & $3.6^{\mathrm{b}} \pm 2.2$ & $2.1 \pm 2.0$ & $53.4 \pm 35.7$ \\
November & 66 & $3.3^{\mathrm{b}} \pm 1.8$ & $1.7 \pm 1.8$ & $46.1 \pm 36.6$ \\
December & 36 & $3.1^{\mathrm{b}} \pm 1.9$ & $1.6 \pm 1.5$ & $55.7 \pm 38.5$ \\
Total Mean & 44 & $3.3^{ \pm} \pm 1.9$ & $1.5 \pm 1.5$ & $44.6 \pm 36.6$ \\
\hline a-b-c Values within a column with different superscripts differ significantly (P<0.05)
\end{tabular}

The general tendency ( $\mathrm{P}<0.02$ ) was for a lower ovarian follicle density (growth) during the warmer (summer) months and after that a higher follicular population during the colder (winter) months. The highest ovarian follicle populations $(\mathrm{P}<0.02)$ were recorded in the months, May and September, and could be associated with seasonal changes.

The number of oocytes recovered, followed the same trend as the follicular population, with the same general tendency of higher numbers of bovine oocytes aspirated during the colder months and lower numbers during the hotter months. There was, however, no significant difference $(P>0.2)$ in the number of oocytes aspirated during the warmer and colder months. The correlation between ovarian follicular population and the number of oocytes recovered amounted to $r=0.79$. 
There were no significant differences $(\mathrm{P}>0.07)$ in recovery rate between the different months of the year. The correlation coefficient recorded between the oocyte recovery rate and follicular population amounted to $r=0.07$. This correlation coefficient was extremely low and it was evident that the oocyte recovery rate decreased with an increase in the follicular population.

The mean ( \pm s.e.) annual oocyte quality recorded in sub-fertile beef cows over a 4-year period is presented in Table 2 .

Table 2 The mean ( \pm s.e.) annual oocyte quality recorded in sub-fertile beef cows over a 4-year period

\begin{tabular}{|c|c|c|c|c|}
\hline \multirow{2}{*}{ Month } & \multirow{2}{*}{$\begin{array}{l}\text { Number } \\
\text { of oocytes }\end{array}$} & \multicolumn{3}{|c|}{ Oocyte quality* (\% of total) } \\
\hline & & Grade A & Grade B & Grade C \\
\hline January & 57 & $47.5 \pm 44.6$ & $49.7 \pm 46.5$ & $2.9 \pm 11.0$ \\
\hline February & 92 & $42.2 \pm 43.6$ & $48.5 \pm 44.3$ & $6.9 \pm 25.8$ \\
\hline March & 61 & $49.9 \pm 46.4$ & $46.8 \pm 46.3$ & $3.5 \pm 18.6$ \\
\hline April & 19 & $75.0 \pm 39.9$ & $16.7 \pm 32.6$ & $8.3 \pm 28.9$ \\
\hline May & 44 & $63.3 \pm 45.0$ & $30.8 \pm 42.7$ & $5.9 \pm 24.5$ \\
\hline June & 85 & $58.2 \pm 40.9$ & $34.7 \pm 41.1$ & $9.8 \pm 23.8$ \\
\hline July & 65 & $61.9 \pm 39.8$ & $32.6 \pm 38.7$ & $5.8 \pm 22.0$ \\
\hline August & 45 & $63.4 \pm 41.0$ & $27.9 \pm 38.2$ & $8.7 \pm 23.0$ \\
\hline September & 50 & $64.3 \pm 45.4$ & $28.6 \pm 43.8$ & $7.1 \pm 23.9$ \\
\hline October & 126 & $60.3 \pm 44.5$ & $39.4 \pm 44.3$ & $0.4 \pm 2.3$ \\
\hline November & 117 & $62.3 \pm 42.3$ & $35.8 \pm 41.3$ & $3.1 \pm 11.7$ \\
\hline December & 60 & $66.1 \pm 41.8$ & $32.2 \pm 42.2$ & $1.7 \pm 9.3$ \\
\hline
\end{tabular}

*Differences not significant.

There was no significant difference $(\mathrm{P}>0.2)$ between the different grades of oocytes classified over the 4-year period. The standard deviation or variation in Grade A oocytes was less pronounced than for the Grade B oocytes and even less than for the Grade C oocytes. There was a tendency for the number of Grade A oocytes to be higher during the cooler months (June to October) and lower during the hotter months (January to March). An inverse tendency was recorded for the Grade B type oocytes. As for the case with the follicle numbers and number of oocytes recovered, the highest Grade A number of oocytes was recorded in April (autumn). The same observation was made for Grade B oocytes - in which case the lowest occurrence was recorded during April. A high negative correlation $(r=-0.95)$ was recorded between Grade $\mathrm{A}$ and $\mathrm{B}$ oocytes.

The mean number of embryos produced per OPU session per cow is presented in Figure 1.

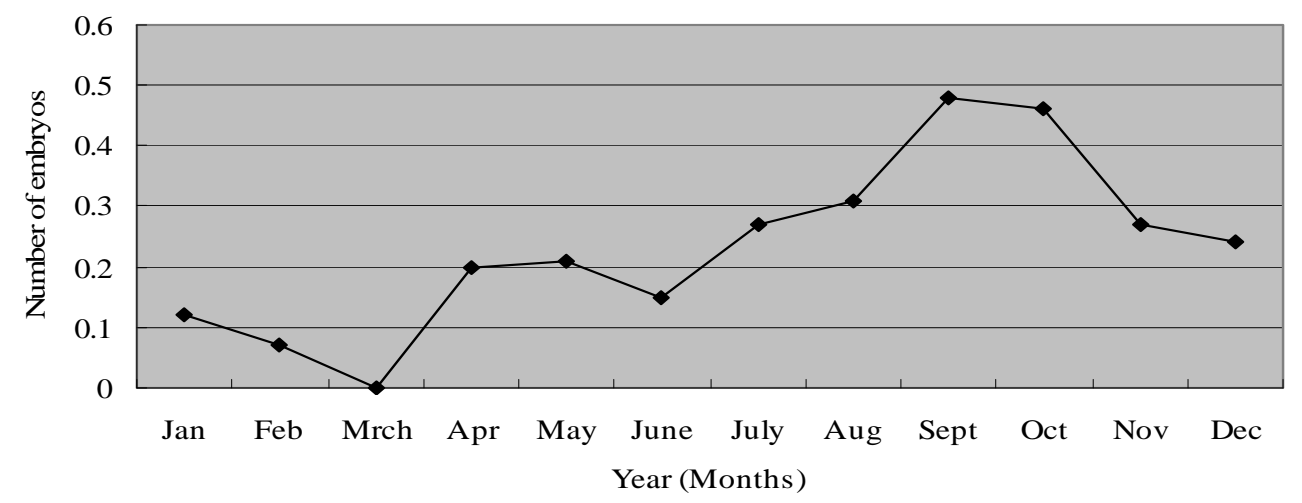

Figure 1 Mean number of embryos produced per oocyte recovery procedure (OPU) session per cow. 
There was no significant difference $(\mathrm{P}>0.2$ ) in the number of embryos produced per OPU session for the different months of the year. The highest mean number of embryos per OPU session was recorded in September $(0.5 \pm 0.9)$ (spring) and the lowest (zero) in March (autumn). The general tendency as determined by this method (embryo production per OPU session per cow) was low, with decreasing embryo production during the warm months, with a steady increase during the cooler months (June to August) - with peak embryo production during spring (September to October). During the entire 4-year period no embryos were produced during the month of March. Although these differences were not significant in statistical terms, it has a big impact on the practical feasibility of embryo production during this specific month of the year. No influence of breed could be detected in terms of embryo production. The correlation recorded between embryos produced per OPU session and Grade A, B and C oocytes was $r=0.56 ; r=-0.47$ and $r=-0.11$ respectively (not significant).

\section{Discussion}

From the results it is evident that there are possibly seasonal trends in aspects such as ovarian follicular populations, the number of oocytes recovered and the number of embryos produced per OPU session. It would seem as if the period between January and March should best be avoided for the application of IVEP procedures in cattle. Although the number of embryos produced is low (15\%) compared to those generated following the use of MOET and IVEP from normally reproducing donors $( \pm 40 \%)$ - it is acceptable due to the fact that these sub-fertile donors could not produce embryos or offspring by any other means than IVEP.

\section{Conclusion}

The effect of season is a very important finding that can have a significant effect on the efficiency of this relatively new technology in the cattle industry. It provides important information which can be used to plan future IVEP programmes for sub-fertile and possibly even reproductively sound donor cows. This strategic planning should also enhance the results obtained in these programmes and to make it more effective for the technology supplier and more cost-effective for the end-user, which is the livestock producer.

\section{References}

Bastidas, P. \& Randel, R.D., 1987. Seasonal effects on embryo transfer results in Brahman cows. Theriogenology 28, 531-540.

Harrison, L.M., Hansen, T.R. \& Randel, R.D., 1982. Evidence for seasonal and nutritional modification of ovarian and pituitary function in crossbred heifers and Brahman cows. J. Anim. Sci. 55, 649-656.

Leibfried, M.L. \& First, N.L., 1979. Characterization of bovine follicular oocytes and their ability to mature in vitro. J. Anim. Sci. 48, 76-86.

Mitchell, L.M., Staines, M.E., McCallum, G.J., Kuran, M. \& McEvoy, T.G., 2000. Season affects in vitro developmental competence of ovine oocytes. Theriogenology 53, 363 (abstract).

Pieterse, M.C., Kappen, K.A., Kruip, T.A. \& Taverne, M.A.M., 1988. Aspiration of bovine oocytes during transvaginal ultrasound scanning of the ovaries. Theriogenology 30, 751-762.

Randel, R.D., 1994. Unique reproductive traits of Brahman and Brahman based cows. In: Factors Affecting Calf Crop. Eds Field, M.J. \& Sand, R.S., Boca Raton, FL: CRC Press. pp. 23-43.

Rhodes, R.C., Randel, R.D. \& Long, C.R., 1982. Corpus luteum function in the Bovine: In vitro and in vivo evidence for both seasonal and breed type effect. J. Anim. Sci. 55, 159-167.

Rutledge, J.J., Monson, R.L., Northey, D.L. \& Leibfried-Rutledge, M.L., 1999. Seasonality of cattle embryo production in a temperate region. Theriogenology 51, 330.

SAS, 1986. Statistical Analysis Systems user's guide. SAS Institute Inc., Raleigh, North Carolina, USA.

Zeitoun, M.M., Rodriquez, H.F. \& Randel, R.D., 1996. Effect of season on ovarian follicular dynamics in Brahman cows. Theriogenology 45, 1577-1581. 